\title{
Photoluminescence and afterglow in samarium-doped zirconia toward biological temperature sensor
}

\author{
Satoshi GOTO ${ }^{1}$, Yoshihiro TAKAHASHI ${ }^{1, \dagger}$, Nobuaki TERAKADO ${ }^{1}$, Yasushi NAKAJIMA ${ }^{2}$, Noriko ONOUE $^{3}$, \\ Tsuyoshi SHINOZAKI ${ }^{3}$ and Takumi FUJIWARA ${ }^{1, \dagger \dagger}$ \\ ${ }^{1}$ Department of Applied Physics, Graduate School of Engineering, Tohoku University, \\ 6-6-05 Aoba, Aoba-ku, Sendai 980-8579, Japan \\ ${ }^{2}$ Daiichi Kigenso Kagaku Kogyo Co., Ltd., 6-38 Hirabayashi Minami 1-chome, Suminoe-ku, Osaka 559-0025, Japan \\ ${ }^{3}$ Department of Cardiovascular Medicine, National Hospital Organization, Sendai Medical Center, \\ 8-8 2-chome, Miyagino, Miyagino-ku, Sendai 983-8520, Japan
}

This study describes the investigation of photoluminescence (PL) and afterglow properties of samarium ion $\left(\mathrm{Sm}^{3+}\right)$-doped zirconia and the comparison with those of pure zirconia. The pure sample exhibits bluish PL and afterglow upon excitation by ultraviolet light, whereas white-to-reddish PL and white afterglow are detected by means of visual observation and spectroscopic measurement in the doped samples. It is therefore suggested that the $\mathrm{Sm}^{3+}$-doped zirconia is applicable to a temperature-sensing probe for the human body because it shows afterglow PL in the near-infrared "biological window" region.

(C2019 The Ceramic Society of Japan. All rights reserved.

Key-words : Zirconia, Photoluminescence, Afterglow

[Received April 15, 2019; Accepted July 8, 2019]

\section{Introduction}

Body temperature is one of the main vital signs of human health and is largely related to physiological functions, including the immune system and metabolism. In addition, because targeted temperature management, formerly called therapeutic hypothermia, has a potential to decrease the risk of brain dysfunction by protecting the brain against head injury and cardiopulmonary arrest, the control and management of temperature are of high relevance in the medical field. ${ }^{1)}$ Therefore, the demand for easy and accurate temperature-sensing techniques applicable to the human body, particularly in deep parts of the living body, has increased. Recently, we proposed a new temperature-sensing technique using nano-phosphor with afterglow photoluminescence (PL) and optically stimulated luminescence (OSL) as a probe. ${ }^{2), 3)}$ The afterglow PL originates from the recombination between a hole and an electron, which are trapped at a metastable site and subsequently released. Because the release of electrons depends on thermal excitation, the environmental temperature determines the PL lifetime. Additionally, the OSL via

Corresponding author: Y. Takahashi; E-mail: takahashi@ laser.apph.tohoku.ac.jp

$\dagger$ Corresponding author: T. Fujiwara; E-mail: fujiwara@ laser.apph.tohoku.ac.jp

tit Preface for this article: Dol http://doi.org/10.2109/jcersj2. 127.P10-1 a near-infrared (NIR) laser enables us to measure the temperature using time and site selectively without any invasive approaches. ${ }^{2), 3)}$

In this proposal, we focused on zirconia, $\mathrm{ZrO}_{2}$, as a temperature-sensing probe because monoclinic $\mathrm{ZrO}_{2}$ exhibits an excellent afterglow property and is a harmless material with high chemical durability. Although $\mathrm{ZrO}_{2}$ possesses a broad afterglow PL with a peak at $\sim 480 \mathrm{~nm}$ after ultraviolet (UV) excitation, ${ }^{4)-7)}$ such wavelength is not compatible with the so-called "biological window" $(\sim 650-1000 \mathrm{~nm})$ in the NIR region. Therefore, to elongate its PL wavelength, we have examined the effect of doping on the PL property of $\mathrm{ZrO}_{2}$. Herein, samarium ion $\left(\mathrm{Sm}^{3+}\right)$ was selected as a dopant because its $f-f$ transitions render it an efficient luminescent center with a wavelength that would allow us to extend the PL of $\mathrm{ZrO}_{2}$ to the NIR region. ${ }^{8)}$

\section{Experimental}

Pure (non-doped) $\mathrm{ZrO}_{2}$ and $\mathrm{Sm}^{3+}$-doped (0.1 and 1 mol \%) $\mathrm{ZrO}_{2}$ (purity: $99.9 \%$, powder state) were provided by Daiichi Kigenso Kagaku Kogyo Co., Ltd. The $\mathrm{ZrO}_{2}$ powders were subjected to thermal treatment at $1400^{\circ} \mathrm{C}$ for $6 \mathrm{~h}$ in an electric furnace under atmospheric condition. The non-doped and doped samples were identified to be monoclinic via a powder $\mathrm{X}$-ray diffraction $(\mathrm{Cu}-\mathrm{K} \alpha)$ analysis, which suggests that the $\mathrm{Sm}^{3+}$ doping did not considerably alter the crystal structure of $\mathrm{ZrO}_{2}$. Visual observation of the usual PL and afterglow PL was conducted using a UV 
Non-doped

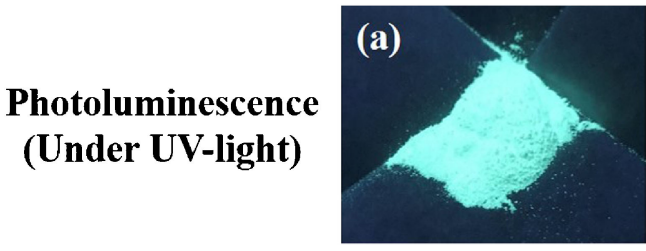

Afterglow
(UV-stopped)

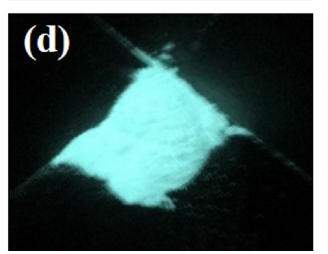

$$
\begin{gathered}
\mathrm{Sm}^{3+} \text {-doped } \\
(0.1 \mathrm{~mol} \%)
\end{gathered}
$$
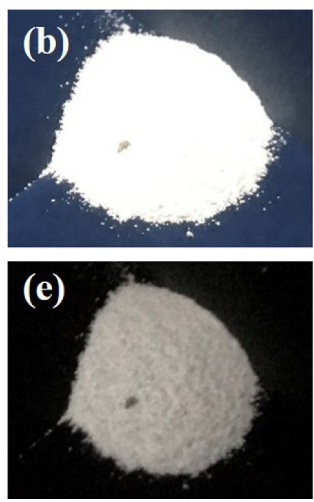

\section{$\mathrm{Sm}^{3+}$-doped \\ (1 $\mathrm{mol} \%)$}
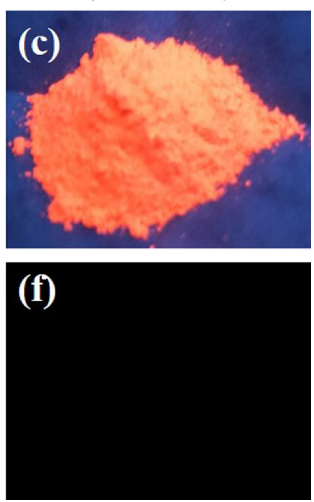

Fig. 1. Non-doped and $\mathrm{Sm}^{3+}$-doped $\mathrm{ZrO}_{2}$ samples under UV irradiation $(\mathrm{a}-\mathrm{c})$ and after stopping the UV light exposure (d-f). The samples without dopant, doped with $0.1 \mathrm{~mol} \% \mathrm{of} \mathrm{Sm}^{3+}$, and doped with $1 \mathrm{~mol}_{\%} \mathrm{of} \mathrm{Sm}^{3+}$ correspond to (a, d), (b, e), and (c, f), respectively. The wavelength of the UV lamp was $254 \mathrm{~nm}$.

lamp at $254 \mathrm{~nm}$. The PL and PL excitation (PLE) spectra were obtained via a spectrofluorometer using a xenon lamp as the excitation source. Moreover, the afterglow PL intensity as a function of time, i.e., the decay curve, was obtained using the spectrofluorometer. In addition, the afterglow PL spectrum was obtained by plotting the afterglow PL intensities, which were observed by excitation at $244 \mathrm{~nm}$, as a function of the monitored wavelengths. All measurements were performed at room temperature.

\section{Results and discussion}

Figure 1 shows the visual PL and afterglow PL features of the non-doped and $\mathrm{Sm}^{3+}$-doped $\mathrm{ZrO}_{2}$ samples under UV light irradiation at $254 \mathrm{~nm}$. In the non-doped sample, visually observable bluish emission and afterglow were confirmed. In addition, white and reddish PLs were observed in the samples doped with 0.1 and $1 \mathrm{~mol} \% \mathrm{Sm}^{3+}$, respectively. The visual reddish emission can be attributed to the $f-f$ transitions of $\mathrm{Sm}^{3+}$ incorporated into the $\mathrm{ZrO}_{2}$ phase. Meanwhile, the sample doped with $0.1 \mathrm{~mol} \% \mathrm{Sm}^{3+}$ exhibited a white afterglow similar to the usual PL, whereas the reddish afterglow was barely observed by the naked eye in the sample doped with $1 \mathrm{~mol} \% \mathrm{Sm}^{3+}$. Thus, it can be concluded that the $\mathrm{Sm}^{3+}$ doping provides a variation in PL coloration and afterglow from blue to red via white.

Figure 2 shows the PLE and PL spectra of the nondoped and $0.1 \mathrm{~mol} \%$-doped $\mathrm{ZrO}_{2}$ samples. For the nondoped sample, the spectral structure of the PLE and PL bands was similar to that previously reported, ${ }^{6}$ ) with a broad band at $\sim 280 \mathrm{~nm}$ in the PLE spectrum and a PL band in the blue region at $\sim 480 \mathrm{~nm}$ in the PL spectrum. Regarding the doped sample $\left(0.1 \mathrm{~mol} \% \mathrm{Sm}^{3+}\right)$, sharp PL peaks in the range of 550-670 $\mathrm{nm}$ [red area in the spectrum (b)] can be observed together with a broad PL band ranging from 400 to $600 \mathrm{~nm}$ (blue area), which is attributable to PL from the host $\mathrm{ZrO}_{2}$ crystal. The sharp peaks were assigned to the $f-f$ transitions of $\mathrm{Sm}^{3+}$ by comparison with the spectroscopic data of previously reported $\mathrm{Sm}^{3+}$-doped

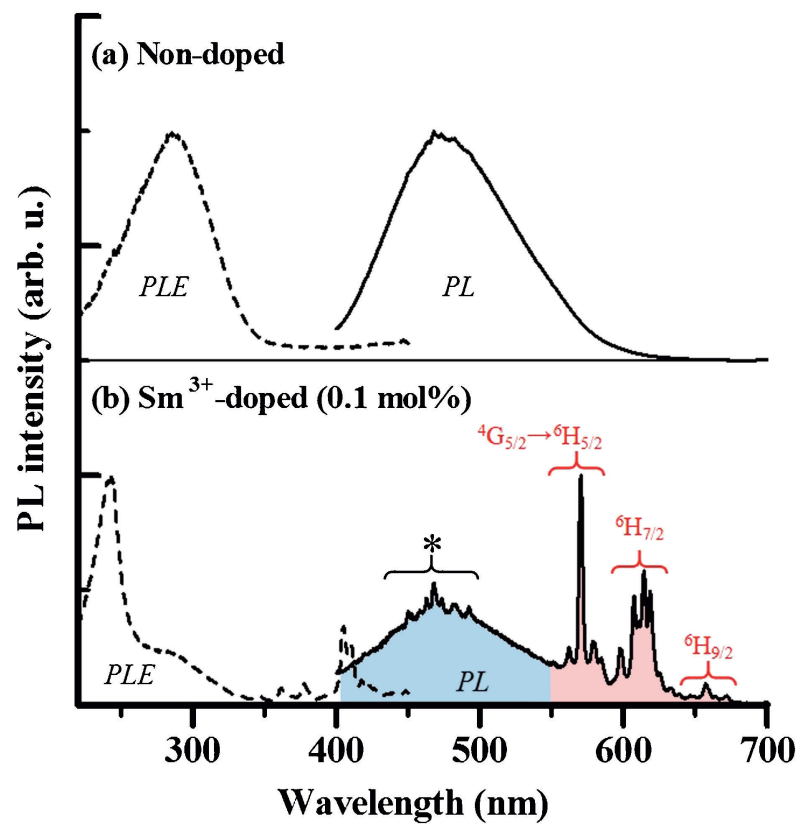

Fig. 2. PLE and PL spectra of the non-doped (a) and $0.1 \mathrm{~mol} \%$ $\mathrm{Sm}^{3+}$-doped $\mathrm{ZrO}_{2}$ (b). The sharp peaks appearing on the broad PL band (marked with an asterisk) in the spectrum of the doped sample are artifacts coming from the xenon lamp used as a light source during the spectroscopic measurement.

crystals. ${ }^{9)-11)}$ According to these data, considering that the variation in PL coloration (Fig. 1; blue-white-red) stems from the combination of PL bands and peaks with different intensities, i.e., reddish PL due to $\mathrm{Sm}^{3+}$ (dopant) and bluish PL owing to $\mathrm{ZrO}_{2}$ (host crystal), appears reasonable. White emission stemming from the combination of PL colors originating in different luminescent centers has also been demonstrated in other materials. ${ }^{12), 13)}$

Figure 3 displays the afterglow PL spectra of the 0.1 mol $\% \mathrm{Sm}^{3+}$-doped sample obtained at different periods (1 and $10 \mathrm{~s}$ after stopping the UV irradiation), together with the usual PL spectrum. In our previous study, the spectral 


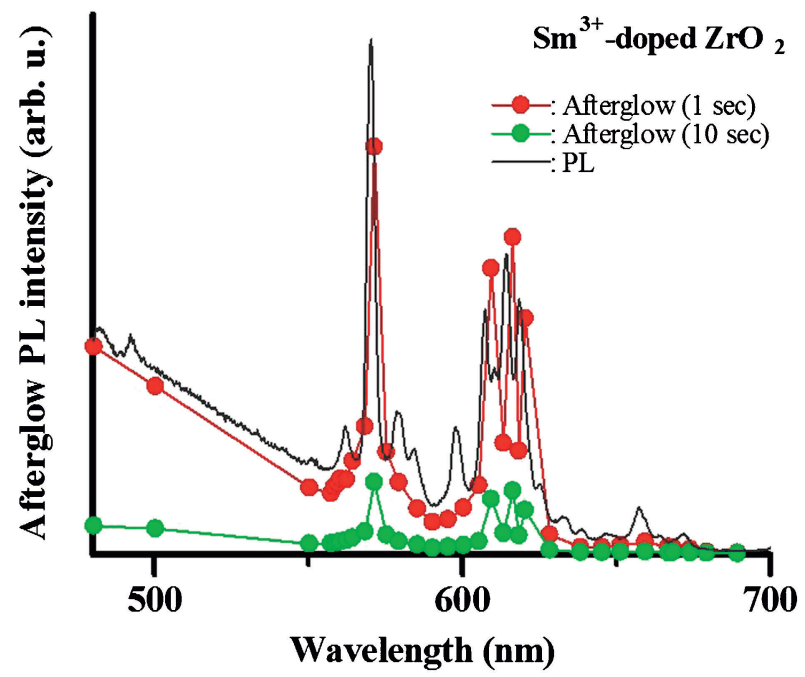

Fig. 3. Afterglow PL intensity as a function of the monitored wavelength, i.e., afterglow PL spectra, of the $0.1 \mathrm{~mol} \% \mathrm{Sm}^{3+}$ doped $\mathrm{ZrO}_{2}$ obtained at 1 and $10 \mathrm{~s}$ after stopping the UV irradiation. The PL data corresponding to Fig. 2(b) are also included.

position/shape of the afterglow PL was nearly superimposed on that of the usual PL of non-doped $\mathrm{ZrO}_{2}$, indicating that the luminescent process/center of afterglow PL was essentially the same as that of the usual PL, i.e., a broad PL band at $400-600 \mathrm{~nm}^{7)}$ In this study, the spectral structure of afterglow PL after $1 \mathrm{~s}$ (plotted by red circles in Fig. 3) was also coincident with that of the PL owing to the $f-f$ transitions of $\mathrm{Sm}^{3+}$ in the $0.1 \mathrm{~mol} \% \mathrm{Sm}^{3+}$-doped $\mathrm{ZrO}_{2}$. Although the afterglow intensity decreased, the spectral structure of afterglow PL after $10 \mathrm{~s}$ (plotted by green circles in Fig. 3) was similar to that after $1 \mathrm{~s}$. This suggests that the PL mechanism of afterglow in the red PL component is totally identical to that of the usual PL, i.e., corresponding to the $f-f$ transitions. Meanwhile, the white afterglow PL can be considered to be due to the combination of afterglow PL originating from the dopant (red) and host crystal (blue).

Figure 4 shows the afterglow decay curves in the nondoped and doped samples. Excitation of the samples was performed by exposure to UV light at wavelengths corresponding to the PLE peak for $10 \mathrm{~min}$. Thus, the detection of afterglow PL intensity was conducted at $480 \mathrm{~nm}$ upon excitation at $284 \mathrm{~nm}$ for the non-doped sample, and the detection and excitation wavelengths were 659 and 244 $\mathrm{nm}$, respectively, for the doped samples. We have already described that the sample containing $0.1 \mathrm{~mol}^{\%} \mathrm{Sm}^{3+}$ showed a white afterglow PL (Fig. 1). The decay curve measurement revealed that afterglow PL occurs in the red region $(659 \mathrm{~nm})$, demonstrating that the rare earth doping of $\mathrm{ZrO}_{2}$ provides an afterglow PL compatible with the biological window. However, the afterglow PL intensity was largely decreased in the $1 \mathrm{~mol} \% \mathrm{Sm}^{3+}$-doped sample, as can be extracted from Fig. 1. Recently, Iwasaki et al. suggested that $\mathrm{V}_{\mathrm{o}}{ }^{*}$ (oxygen vacancies with charges of +2 in Kröger-Vink notation) and $\mathrm{Zr}^{2+}$ are related to the meta-

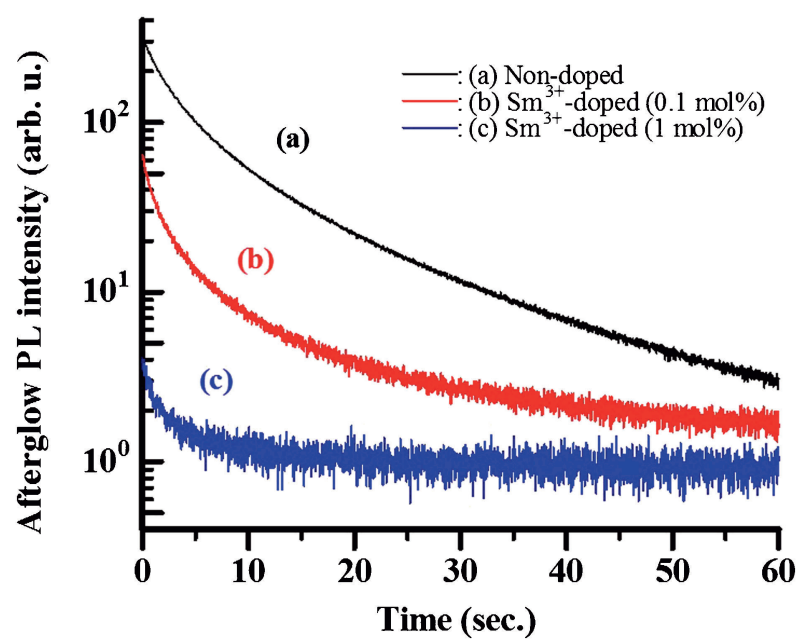

Fig. 4. Afterglow decay curves of the non-doped and $\mathrm{Sm}^{3+}$ doped $\mathrm{ZrO}_{2}$. The decay curves were obtained by the detection of afterglow PL intensity at $480 \mathrm{~nm}$ upon excitation at $284 \mathrm{~nm}$ for the non-doped sample (a). The detection and excitation wavelengths were 659 and $244 \mathrm{~nm}$, respectively, for the doped samples (b) and (c). The UV-exposure time for the excitation was $10 \mathrm{~min}$.

stable sites in $\mathrm{ZrO}_{2}{ }^{7}{ }^{7}$ Therefore, we tentatively conclude that the incorporation of an excess amount of $\mathrm{Sm}^{3+}$ into $\mathrm{ZrO}_{2}$ suppresses the formation of metastable trapping sites. Therefore, a further study involving detailed spectroscopic analysis and consideration of defect formation is necessary to gain a deeper understanding of the suppression of the afterglow property.

\section{Summary}

We have examined the PL and afterglow properties of non-doped and $\mathrm{Sm}^{3+}$-doped monoclinic $\mathrm{ZrO}_{2}$ on the basis of the optical measurements. The $\mathrm{ZrO}_{2}$ powder doped with $0.1 \mathrm{~mol} \%$ of $\mathrm{Sm}^{3+}$ exhibited white PL and afterglow PL upon UV excitation, which can be attributed to the combination of bluish PL from the host crystal and reddish PL stemming from the $f-f$ transitions of the dopant. The afterglow PL spectral and decay curve measurements suggest that the addition of a suitable dopant provides the required elongation of afterglow PL wavelength that ensures its compatibility with the biological window.

Acknowledgments This study was supported by the Ministry of Education, Culture, Sports, Science and Technology of the Japanese Government. The authors would like to thank Mr. Masaharu Ohashi of Tohoku University for remarkable contributions to this study.

\section{References}

1) N. Onoue, Sendai Med. Center J., 2, 25-36 (2012) [in Japanese].

2) Y. Takahashi, N. Terakado, N. Onoue, T. Shinozaki and T. Fujiwara, J. Soc. Inorg. Mater. Jpn., 24, 415-421 (2017) [in Japanese].

3) Y. Takahashi, N. Terakado, N. Onoue, T. Shinozaki and T. Fujiwara, Ceram. Japan, 53, 500-502 (2018) [in Japanese]. 
4) J. F. Sarver, J. Electrochem. Soc., 113, 124-128 (1966).

5) Y. Nobuta, Y. Takahashi, T. Miyazaki, N. Terakado, N. Onoue, T. Shinozaki and T. Fujiwara, J. Ceram. Soc. Jpn., 125, 343-347 (2017).

6) Y. Nobuta, Y. Takahashi, T. Miyazaki, N. Terakado, N. Onoue, T. Shinozaki and T. Fujiwara, J. Non-Cryst. Solids, 501, 49-54 (2018).

7) K. Iwasaki, Y. Takahashi, N. Terakado, N. Onoue, T. Shinozaki and T. Fujiwara, Ceram. Int., 44, 1292912933 (2018).

8) M. Tamatani, "Phosphor Handbook, 2nd Edition", Ed. by W. M. Yen, S. Shionoya and H. Yamamoto, CRC Press, Boca Raton (2007) pp. 196-197.
9) B. Yan and X. Xiao, J. Alloy. Compd., 433, 251-255 (2007).

10) P. K. Nayak, W. J. Kao, D. Sahu, J. L. Huang, D. Sahu and J. L. Huang, J. Am. Ceram. Soc., 93, 3481-3485 (2010).

11) S. Ponkumar, K. Janaki, D. Prakashbabu, H. B. Ramalingam, K. Munirathnam, T. V. M. Sreekanth and S. J. Dhoble, Appl. Phys. A-Mater., 124, 134 (2018).

12) M. Kinoshita, Y. Takahashi, N. Terakado, H. Masai and T. Fujiwara, J. Ceram. Soc. Jpn., 123, 611-614 (2015).

13) Y. Hoshino, Y. Takahashi, N. Terakado and T. Fujiwara, Jpn. J. Appl. Phys., 56, 120302 (2017). 